\title{
A Lightweight Approach to 3D Measurement of Chronic Wounds
}

\author{
Tim Shirley, Dmitri Presnov, Andreas Kolb \\ Computer Graphics Group,University of Siegen, Hoelderlinstrasse 3, 57076 Siegen \\ tim.shirley@student.uni-siegen.de, \{dmitri.presnov,andreas.kolb\}@ uni-siegen.de
}

\begin{abstract}
This paper presents a light-weight process for 3D reconstruction and measurement of chronic wounds using a commonly available smartphone as an image capturing device. The first stage of our measurement pipeline comprises the creation of a dense 3D point cloud using structure-from-motion (SfM). Furthermore, the wound area is segmented from the surrounding skin using dynamic thresholding in CIELAB color space and a surface is estimated to simulate the missing skin in the wound area. Together with a mesh reconstruction of the wound, the skin surface and the segmented wound is used to calculate the wound dimensions, i.e., its length, surface area and volume. We evaluate the presented pipeline using three wound phantoms, representing different stages in healing, and compare the subsequently scanned and measured wound dimensions with manually measured ones.
\end{abstract}

\section{Keywords}

Scene Reconstruction, Wound Measurement, Structure-from-Motion, Object Segmentation.

\section{INTRODUCTION}

Chronic wounds are a major and growing health issue worldwide. Besides increasing mortality and treatment expenses, they cause substantial pain and distress due to, e.g., significantly reduced mobility, lower selfesteem and social isolation [1]. As 1-2\% of the population $[1,2]$ are affected by chronic wounds, they are also considered a "silent epidemic". Since chronic wounds mainly affect elderly patients, the demographic shift within Western societies causes their increasing dissemination [3].

The most common type of chronic wounds are venous and arterial ulcers, which primarily affect elderly patients. Diabetic ulcers, one of the characteristics of the diabetic foot syndrome and a frequent long-term result of diabetes mellitus, are also very common. Immobile or paralyzed patients often develop pressure ulcers, which are caused by restricted blood flow due to prolonged pressure between repositioning intervals. The tropical disease leishmaniasis, transmitted by the bite of the female sandfly, is another major cause of cutaneous chronic wounds [4].

Evidently, 3D wound measurement approaches are advantageous as they offer much more insight into wound

\footnotetext{
Permission to make digital or hard copies of all or part of this work for personal or classroom use is granted without fee provided that copies are not made or distributed for profit or commercial advantage and that copies bear this notice and the full citation on the first page. To copy otherwise, or republish, to post on servers or to redistribute to lists, requires prior specific permission and/or a fee.
}

healing, e.g. for deep ulcers, where much of the early healing progress takes place at the bottom of the wound bed [5]. Therefore, an affordable, light-weight 3D wound capturing system has a lot of potential, especially against the background of a growing number of elderly patients and the requirement to provide treatment in underdeveloped and/or rural regions [4].

Wound healing is a highly complex process and the treatment of chronic wounds requires close monitoring over a long time period, sometimes years. Besides careful qualitative observation and documentation by medical staff, reliable quantitative measurements are very important in order to monitor the wound's healing, i.e. its change in size and shape over time. Numerous different measurement techniques exist, ranging from very simple ruler-based size estimates to advanced multisensor 3D systems utilizing state of the art computer vision algorithms. However, simple methods are unreliable, imprecise, and uncomfortable for the patient, while advanced systems are often expensive, inefficient to use, and no gold standard has yet been established for wound measurement so far [6].

Current computer vision based approaches either use stereo vision [7], also available in commercial systems like MAVIS $I I^{1}$ or Time-of-Flight (ToF) measurements [8]. However, stereo imaging as well as ToF range measurement requires specific camera devices and neither stereo nor ToF can be considered ubiquitous sensors, so far. Furthermore, compared to current RGB cameras in mobile phones, ToF suffers from low

\footnotetext{
1 imaging.research.southwales.ac.uk/
} projects/wm/mavis 
image resolution and from depth measurements errors due to subsurface scattering [9].

In this paper, we present a light-weight wound measurement system based on RGB images captured using standard smartphone cameras, thus solving the wound measurement problem within the emerging domain of mobile health (mHealth) [10]. The integrated sensors in smartphones deliver images of decent quality, allowing for high quality photography at very low cost.

Our 3D wound reconstruction pipeline comprises Structure-from-Motion (SfM) as monocular 3Dreconstruction technique. The further processing of the resulting sparse point clouds involves a segmentation into skin and wound regions, the extraction of the wound's contour, and a mesh reconstruction of the wound. Based on the resulting 3D reconstructions of wound and a surface fitting of the skin in order to estimate the healthy state, our approach performs automatic length, area and volume measurements. For evaluation, we produced a sequence of wound phantoms for which we acquired several series of images.

\section{PRIOR WORK}

Mukherjee et al. [11] give an overview on contact-free, sensor-based techniques for wound measurement, discussing optical approaches as well as approaches that involve more exhaustive sensors such as hyperspectral, thermal and laser doppler imaging or confocal microscopy. While $2 D$ wound measurement approaches have been developed for more than two decades and are still being researched $[12,13]$, this paper focuses on optical approaches for $3 D$ wound measurement.

The idea of using standard 2D RGB imagery for 3D wound reconstruction and measurement dates back more than one decade. These early approaches reconstruct sparse 3D point clouds from stereo or multi-view imagery taken from the wound. Albouy et al. [14] use a Harris corner detector, cross-correlation, outlier removal and homography estimation in order to compute a sparse $3 \mathrm{D}$ point cloud that is used for estimating the wound's volume. Treuillet et al. [5] use stereophotogrammetry in order to reconstruct a sparse 3D point cloud. More recently, general approaches for 3D geometry reconstruction using 2D images acquired with smartphones have been proposed $[15,16]$. While Kolev et al. [15] create a point-based 3D model by integrating multiple stereo-based depth hypotheses into a compact and consistent 3D model, Muratov et al. [16] use an SfM like approach that involves additional IMU data. Sirazitdinova and Deserno [7, 17] propose to use similar approaches in 3D wound assessments. They opt for motion stereo [18] in order to reconstruct 3D geometry from 2D RGB imagery acquired with a smartphone. So far, there is no publicly available documentation regarding the system's implementation or evaluation.

Gaur et al. [8] describe an alternative 3D wound measurements system based on RGB-D imagery captured with an Intel ToF camera. After registration of the RGB and the depth images, the wound is segmented directly in image space using standard filter and morphological operators. Then, they identify the wound's boundary pixels and fit a plane to the boundary's range data. Both, the wound and the estimated healthy skin are modeled as quadratic surfaces, in order to measure the wound's volume.

Summary. Current 3D wound measurement approaches mainly rely on special-purpose hardware for wound acquisition, such as stereo vision or time-of-flight cameras. While early approaches, involving 2D RGB images, deliver only sparse $3 \mathrm{D}$ geometric information and are therefore rather inaccurate, there are no reports on successfully applying recent improvements in dense 3D scene reconstruction from RGB images on mobiles to 3D wound measurement. In this paper we present an SfM pipeline that can be successfully applied to 3D wound reconstruction.

\section{METHOD}

In this section, we present our light-weight wound measurement system. Our approach uses a sequence of RGB images of a chronic wound that has been captured using standard smartphone cameras. Fig. 1 depicts the main components of our wound measurement system that can be summarized as follows:

Acquisition: A sequence of images or a video is taken from the considered chronic wound. Additionally, a marker is located close to the wound in order to solve for the scale ambiguity inherent to SfM surface reconstruction.

Structure from Motion (SfM): Based on features extracted from the input images, image pairs with large overlap are identified, camera poses are estimated and a 3D point cloud is computed (see Sec. 3.1).

Point Cloud Preprocessing: Depending on the input imagery, the resulting raw point cloud contains a significant number of outliers that are removed in this stage. Furthermore, the scale ambiguity is resolved using the marker that has been placed in the scene (see Sec. 3.2).

Wound Segmentation \& Fitting: The clean point cloud is segmented in wound and skin using a color thresholding and clustering. Furthermore, the contour of the wound is extracted and a surface is fitted to the skin region, which represents the condition of healthy skin (see Sec. 3.3). 


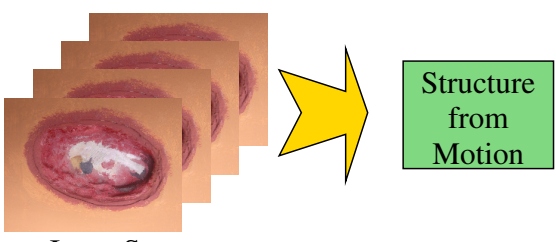

Input Sequence
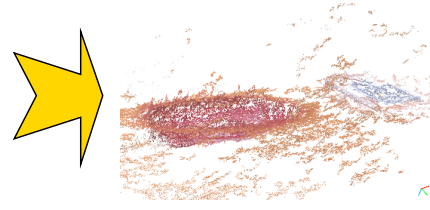

Raw Point Cloud
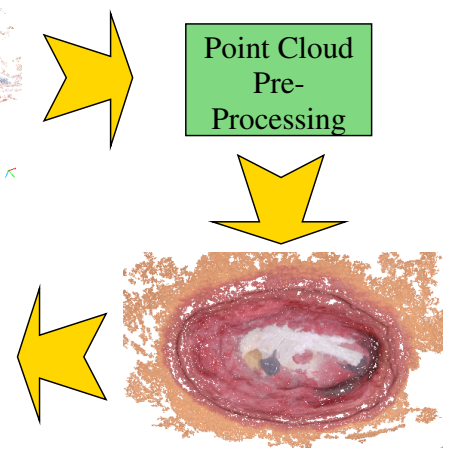

Clean Point Cloud
Contour \& Surface Fitting

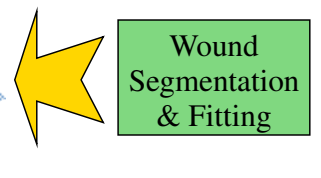

gmentation

Wound
Measurement

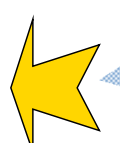

Figure 1: Overview of the proposed 3D wound reconstruction method.

Wound Measurement: In this stage the segmented wound points are converted into a mesh. Based on the meshed wound region, the wound's size, area and volume are deduced (see Sec. 3.4).

\subsection{Structure from Motion (SfM)}

Structure-from-Motion (SfM) is a well established technique from scene reconstruction that uses monocular RGB image sequence as input. This section briefly describes the main concept behind SfM based on Schoenberger and Frahm [19]. The SfM process consists of two main stages, correspondence finding and incremental reconstruction.

Correspondence Finding. Given a set $\left\{I_{i} \mid i=1 \ldots N_{I}\right\}$ of unordered images, feature sets $\mathscr{F}_{i}=\left\{\left(\mathbf{x}_{j}, \mathbf{f}_{j}\right) \mid j=\right.$ $1 \ldots N_{F_{i}}$ \} representing features $\mathbf{f}_{j}$ at image locations $\mathbf{x}_{j}$ are extracted for each image $I_{i}$. Frequently, the scaleinvariant feature transform (SIFT) is used [20]. Based on the feature sets $\mathscr{F}_{i}$, image pairs with sufficient spatial overlap, i.e. with sufficient common features are identified using, e.g., the Lukas-Kanade tracker [21]. Afterwards, mismatching feature correspondences are removed using RANSAC, and the pairwise image transformations are established.

Incremental Reconstruction. The scene model is described by a set of points $\mathscr{X}$ and a set of camera poses $\mathscr{P}$. SfM is initialized with a carefully selected image pair and its reconstructed 3D points using triangulation. Further images are registered using the feature correspondences. As the incremental nature of this approach and the inherent imprecision of point estimates causes accumulation of errors and point drift, the reconstruction parameters, i.e., the camera poses $\mathscr{P}$ and the $3 \mathrm{D}$ point locations $\mathscr{X}$ are regularly refined using bundle adjustment [22]. This process minimizes the reprojection error applied to each $3 \mathrm{D}$ point $\mathbf{X}_{j}^{\text {corr }} \in \mathscr{X}$ corresponding to the feature location $\boldsymbol{x}_{j}$ in image $I_{i}$, i.e.

$$
E=\sum_{i=1}^{N_{I}} \sum_{j=1}^{N_{F_{i}}} \rho\left(\left\|\pi\left(\mathbf{P}_{i}, \mathbf{X}_{j}^{\text {corr }}\right)-\mathbf{x}_{j}\right\|_{2}^{2}\right)
$$

where $\pi$ is the projection function defined by the camera pose $\boldsymbol{P}_{i} \in \mathscr{P}$ for image $I_{i}$ and $\rho$ is a loss function. This results in a non-linear optimization process.

\subsection{Point Cloud Preprocessing}

As the SfM method is intrinsically scale ambiguous, wound measurement requires proper rescaling of the scene, i.e. of the point cloud reconstructed by SfM. Therefore, we place a $30 \times 30 \mathrm{~mm}$ ArUco marker [23] that is printed on a $45 \times 45 \mathrm{~mm}$ board close to the wound. Due to the high contrast of the marker, the marker's points can be segmented easily using fixed color thresholds. Afterwards, a plane is fitted to the marker points using RANSAC and the scene is transformed into the marker's plane. Then, the marker dimensions are determined and the point cloud is rescaled using the resulting isotropic scale factor. Finally, the marker region is removed from the point cloud using a simple spatial cropping.

Although SfM produces dense and comparably precise point clouds, they frequently contain patches of outlier points that are caused by faulty correspondences due to, for example, specular reflections in the input images. Most outliers form small clusters or spurious sheets disjunct from the main point cloud representing the captured object. Therefore, we apply a clustering based on the density-based algorithm proposed by Ester et al. [24].

\subsection{Wound Segmentation, Surface Fitting \& Meshing}

In order to perform wound measurement, the wound must be segmented from the surrounding skin. As the wound's boundary does not necessarily have distinct geometric features, we opt for segmentation using color features. Therefore, we transform the point colors into the CIELAB color space since it emphasizes the color difference between healthy skin and wound tissue [25] (see Fig. 2a). The color disparity in the $b^{*}$-channel allows for a clear distinction between wound and skin using a simple dynamic threshold applied to the histogram 


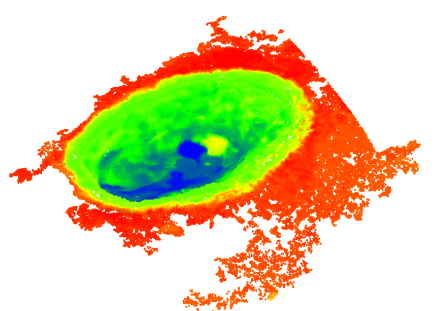

(a) Color mapped CIELAB $b^{*}$ channel

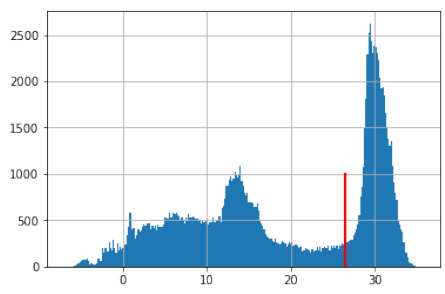

(b) Histogram with threshold

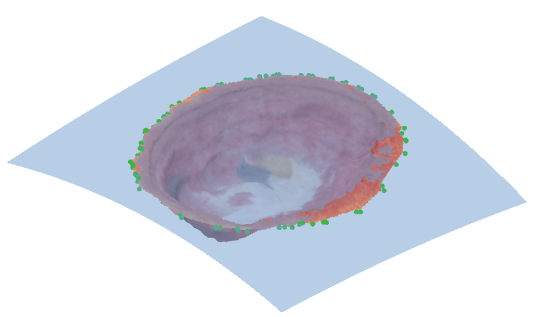

(c) Surface fitting \& contour

Figure 2: Segmentation and surface fitting: The color mapped visualization of the CIELAB $b^{*}$ channel (2a), the histogram with threshold for segmentation $(2 b)$, and the finally segmented wound points with the contour points (green) and the fitted skin surface (2c).

of all colors in the point cloud using the triangle algorithm [26] (see Fig. 2b).

Color segmentation may result in spurious points that have been wrongly classified. This mainly happens in the wound region in which points occasionally are classified as skin. In order to remove residual clusters or falsely classified points in either region, we extract the largest connected skin and wound region using the clustering approach from Ester et al. [24].

After segmentation, we identify the points on the wound's contour. Therefore, we compute the 4neighborhood for each point and select the points as contour, if two of their neighbors are classified as skin and two as wound. The resulting set of contour points is coarsened by subsampling. To this end, we use a coarse $16^{3}$ voxel grid and select in each cell the contour point closest to the voxel center. Furthermore, we apply a statistical contour smoothing approach by analyzing the discrete curvature of each contour point $\boldsymbol{c}_{i}$ defined as $\operatorname{curv}\left(\boldsymbol{c}_{i}\right)=\frac{\left\|\boldsymbol{c}_{i+1}-2 \cdot \boldsymbol{c}_{i}+\boldsymbol{c}_{i-1}\right\|}{\boldsymbol{c}_{i+1}-\boldsymbol{c}_{i-1}}$. Calculating the mean $\mu$ and standard deviation $\sigma$ of the curvature values, we remove all contour points above one sigma, i.e. with $\operatorname{curv}\left(\boldsymbol{c}_{i}\right)>\mu+\sigma$.

Next, we apply least square fitting to extract two surfaces, i.e. a planar surface to the contour points and a quadratic surface to the points classified as skin. The latter resembles the healthy state of the wound region. Both surfaces are utilized in the subsequent wound measurement (see Sec. 3.4 and Fig. 2c).

Lastly, the wound's point cloud is transferred into a triangular mesh using Poisson surface reconstruction [27]. This is necessary in order to perform a volume measurement of the wound (see Sec. 3.4).

\subsection{Wound Measurement}

Based on the reconstructed mesh, the segmentation of the points into subsets $\mathscr{S}$ for skin, $\mathscr{C}$ for contour and $\mathscr{W}$ for wound, and the fitted planar surface (w.r.t. the contour points) and quadratic surface (w.r.t. the skin points), we extract the wound's length, area and volume.
The length of the wound is simply the maximum distance between contour points, i.e. $\max \{\|\mathbf{x}-\mathbf{y}\| \mid \mathbf{x}, \mathbf{y} \in$ $C\}$. The area is computed by projecting the contour points $\mathscr{C}$ onto the planar surface resulting in $\mathscr{C}^{\prime}$. Calculating the center of gravity $\mathbf{g}$, the area is computed by summing up the area of all triangles $\triangle\left(\mathbf{c}_{1}, \mathbf{c}_{2}, \mathbf{g}\right)$ formed by any two adjacent contour points $\mathbf{c}_{1}, \mathbf{c}_{2}$ and the center of gravity [28].

In principle, the wound's volume is enclosed by the reconstructed surface mesh including the wound and contour points, and the triangulated quadratic surface that estimate the healthy skin. However, both geometries do not perfectly intersect at the wound's contour line. Creating a single, closed mesh is a non-trivial task, as direct mesh intersection and hole filling may not lead to a proper solution, e.g. to spurious volume fractions, in case of non-planar contours. Instead, we use the reference plane generated from the contour points and compute the wound's volume based on the height fields $s(\boldsymbol{x}), w(\boldsymbol{x})$ of the skin and the wound surfaces parameterized above the reference plane, respectively. Using the triangulated wound surface, the volume is calculated as

$$
\sum_{\triangle \in \mathscr{W}} \cos (\phi) \cdot \operatorname{area}(\triangle) \cdot\left(s\left(\boldsymbol{c}_{\triangle}\right)-w\left(\boldsymbol{c}_{\triangle}\right)\right),
$$

where $c_{\triangle}$ is the center of the triangle in the parameter plane and $\phi$ is the angle between the triangle normal and the normal of the reference plane. We make sure to consider only wound regions that lie within the wound's contour in the reference plane.

\section{IMPLEMENTATION}

The pipeline presented in Sec. 3 has been implemented as prototype on a PC/laptop environment in order to verify its proper operation. We use the COLMAP approach that incorporates the SfM optimizations presented by Schoenberger and Frahm [19] and their multiview-stereo approach [29]. To enable a fast and flexible development of the further pipeline stages, the programming language python together with the scipy [30] 


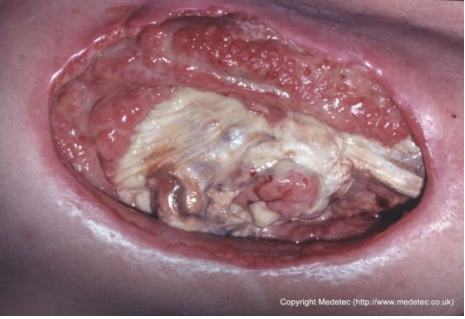

(a) Large pressure ulcer

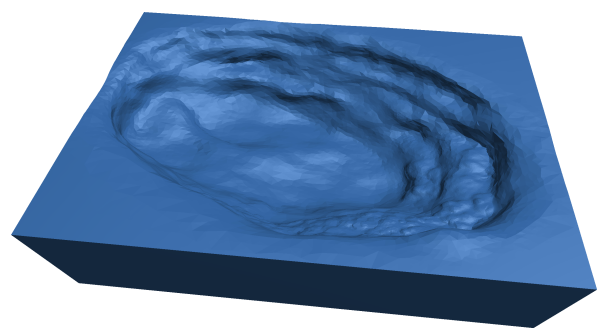

(b) 3D ulcer model

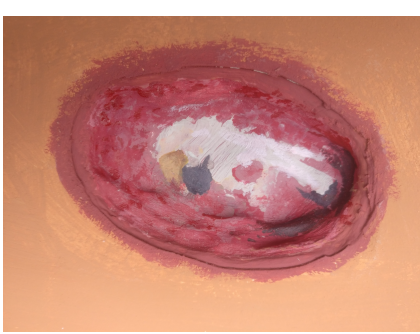

(c) The final phantom

Figure 3: Creating the wound phantom: The reference photograph of a large pressure ulcer (a), the 3D model sculpted in blender (b), and the final painted clay model created using a 3D printed mold (c).

software package was selected. The pyntcloud ${ }^{2}$ library as well as the pandas data structure [31] was used for most point cloud processing operations. For effective and efficient code development, the entire pipeline, with the exception of the Poisson surface reconstruction, has been integrated into a jupyter notebook ${ }^{3}$. For color CIELAB-based segmentation we used the skimage library [32] that provides the function for color transformation and dynamic thresholding.

\section{RESULTS}

\subsection{Phantom Creation \& Manual Wound Measures}

Unfortunately, there are no publicly available ground truth data sets for chronic wounds. Therefore, we fabricated wound phantoms based on an available photograph of a pressure ulcer ${ }^{4}$ that was sculpted using the $3 \mathrm{D}$ modeling software blender $^{5}$. A negative of the digital model was 3D-printed and used as a mold to create physical models with modeling clay. The clay was subsequently painted to roughly resemble the wound photograph and glued to a bent cardboard. Based on the first model resembling the photograph, two further wound models were created, simulating healing and shrinking of the wound area and volume. Thus, we finally have three wound phantoms Large, Medium, and Small. For scale disambiguation, a marker was placed in the scene (see Sec. 3.2).

We manually determine reference values for the wound measures. The length measurements were taken with a household tape measure at one millimeter precision. The area was determined by placing a sheet of millimeter paper on the wound, tracing the contour with a pen and manually reading the area. Both approaches for manually estimating the length and the area of the wound are frequently used in clinical practice. The

\footnotetext{
2 github.com/daavoo/pyntcloud

3 jupyter.org

4 www.medetec.co.uk/slide\%20scans/

pressure-ulcer-images-a/target 92 .html

5 www.blender. org
}

wound volume was determined by filling the phantom with water using a $0.1 \mathrm{ml}$ precision syringe. All three manual reference measurements have been conducted by a student with background in medical informatics w/o any practical experience in wound measurement.

\subsection{Image Acquisition}

In order to assess the quality of the reconstruction pipeline, we acquired three image sequences using a OnePlus 3 (A3003) smartphone with a Sony IMX298 16 megapixel CMOS sensor paired with a F/2.0 aperture lens and phase detection focus. For each wound phantom, we acquired two sets of images (@ $4640 \times 3480$ resolution) and one video sequence (@ $1920 \times 1080$ resolution). The Img_Fast image sequence was captured within 30s, while the Img_Acc image sequence focuses on the precise image acquisition. The video sequence Video is approximately 30s long, captured at some 5 frames per second. Tab. 1 states the precise number of images in column \#im).

\subsection{Evaluation}

\section{D Reconstruction Quality}

Since the precise geometry of the clay wound phantom is unknown and no alternative (or gold standard) 3D reconstruction method was available, we directly evaluated the quality of the $3 \mathrm{D}$ reconstruction by comparing the clean point cloud produced by our SfM method to the original 3D wound model created with blender. Thus, the resulting error also incorporates imprecision introduced by the 3D printing and the molding process. The differences between the reconstructed point clouds and the original 3D models were calculated with CloudCompare $^{6}$. Tab. 1 depicts the resulting geometry error in column geom.err. We find a very good agreement of the reconstruction with respect to the digital wound model of a standard deviation below $1 \mathrm{~mm}$. Note, that the mean error is close to zero, as the reconstructed point clouds and the original 3D models are co-registered for comparison.

$6 \overline{\text { wwW. cloudcompare.org }}$ 

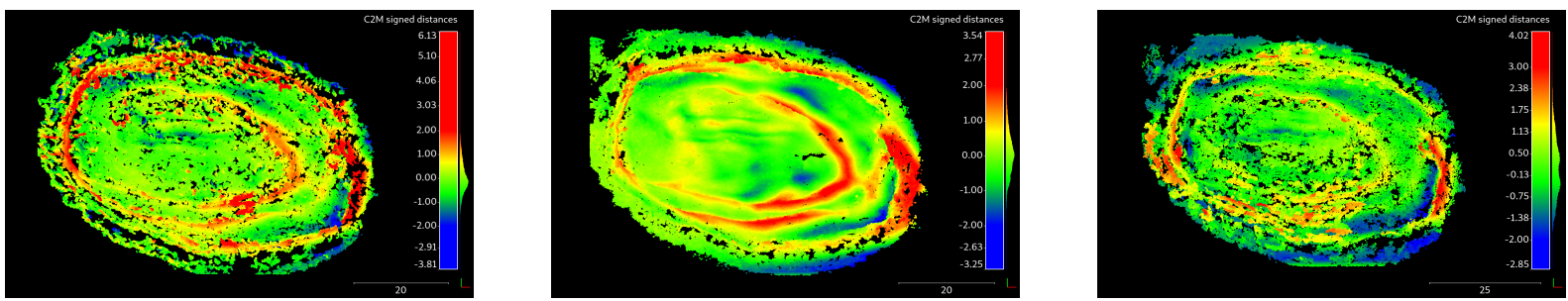

Figure 4: 3D Reconstruction Quality. Color-coded geometric error of the reconstructed wound Medium for the image sequence Img_Fast (left), Img_Acc (middle), and Video (right).

\begin{tabular}{l|r|r|r|r|r|r|r|r|r|r|r|r} 
Wound/Sequ. & \multirow{2}{*}{ \#im } & \multicolumn{2}{|c|}{ \#recon.points } & \multicolumn{2}{|c|}{ scale } & \multicolumn{2}{|c|}{ geom.err. [mm] } & \multicolumn{2}{|c|}{ segm.qual. [\%] } & \multicolumn{3}{|c}{ meas.comp. [\%] } \\
& & raw & clean & acc[\%] & mean & std & TPR & TNR & ACC & $l$ & $A$ & $V$ \\
\hline Large/Img_Fast & 13 & 232,213 & 208,149 & 86.5 & 0.06 & 0.58 & 99.6 & 82.4 & 94.9 & 6.3 & -46.1 & -45.6 \\
Large/Img_Acc & 50 & 768,990 & 581,003 & 98.0 & 0.04 & 0.61 & 99.8 & 89.8 & 96.7 & -2.1 & -10.9 & -10.1 \\
Large/Video & 154 & 158,119 & 108,892 & 99.9 & 0.05 & 0.71 & 94.2 & 88.7 & 92.0 & -1.4 & -11.9 & -14.3 \\
\hline Medium/Img_Fast & 30 & 536,395 & 337,581 & 98.0 & 0.10 & 0.91 & 96.8 & 86.1 & 92.5 & -1.1 & -10.3 & -9.8 \\
Medium/Img_Acc & 50 & 924,083 & 810,568 & 100.1 & 0.09 & 0.76 & 99.6 & 92.1 & 96.0 & 4.5 & -12.9 & -20.6 \\
Medium/Video & 150 & 250,629 & 207,327 & 93.4 & 0.10 & 0.93 & 95.5 & 89.8 & 92.5 & 3.8 & -8.6 & -16.4 \\
\hline Small/Img_Fast & 50 & 401,751 & 322,663 & 93.1 & 0.04 & 0.44 & 99.0 & 91.3 & 95.6 & 11.4 & -3.6 & 2.2 \\
Small/Img_Acc & 50 & 986,796 & 486,801 & 99.3 & 0.15 & 0.80 & 99.4 & 90.5 & 95.4 & 4.4 & -17.7 & -20.2 \\
Small/Video & 143 & 286,819 & 224,004 & 97.3 & 0.12 & 0.12 & 93.5 & 93.6 & 93.6 & 3.3 & -16.0 & -18.0
\end{tabular}

Table 1: Qualitative results for all three wound phantoms and all three image sequences captured for each phantom. The columns contain the number of images in the sequences (\#im), the number of reconstructed points before and after outlier removal and cropping (\#recon.points), the accuracy of the geometric scale factor (scale acc), the geometric error w.r.t. the digital wound model (geom.err.), the segmentation qualitative (segm.qual.), and the wound measurement comparion w.r.t. the manually deduced wound measures (meas.comp.).

Furthermore, Fig. 4 shows the color-coded geometric reconstruction error for geometric error of the reconstructed wound Medium for all three image sequences. As to be expected, our approach reconstructs more points in case of the accurate image sequence. However, the video acquisition yields comparable results regarding the geometric error.

Ignoring the Large/Img_Fast experiment, which will be discussed below, the scale factor has been determined at least with $93 \%$ accuracy (see Tab. 1, col. \#scale acc). Furthermore, 50-90\% of the reconstructed points have been finally segmented as part of the skin or the wound, i.e. they have passed outlier removal and the cropping of the marker (see Tab. 1, col. \#recon.points).

\section{Segmentation Quality}

Consulting the segmentation quality (column segm.qual. in Tab. 1), we find that for all wound phantoms and all image sequences our approach achieves good to very good results. The true positive rate (TPR) is $93.5-99.8 \%$, the true negative rate (TNR) is in the range of $82.4-93.6 \%$, and the accuracy is between $92 \%$ and $96.7 \%$.

\section{Wound Measurement Quality}

Tab. 1, column meas.comp., depicts the wound measurement comparison to the manually deduced wound

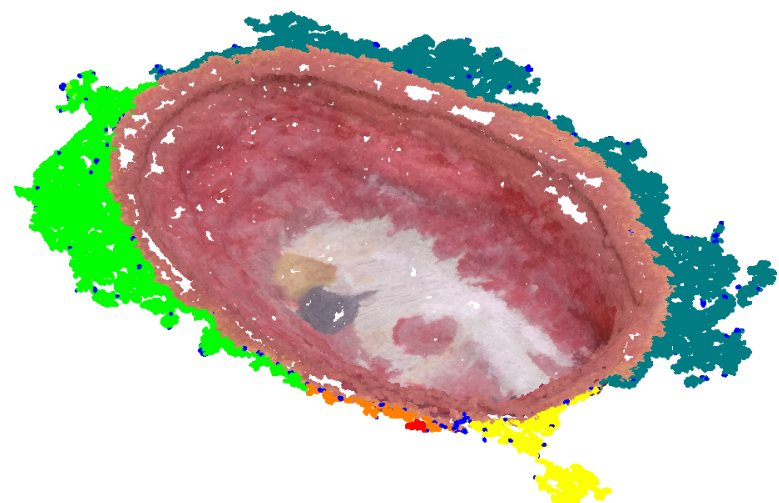

Figure 5: Segmentation result for the skin region of the experiment Large/Img_Fast.

measures. First, we will discuss the results except for Large/Img_Fast, for which we get significant larger errors than for the other experiments. Compared to the manual measurement, the wound's length has been estimated fairly accurate, only the Small/Img_Fast experiment results in an error of $11 \%$. In general, the length is slightly over-estimated. Regarding the wound area, our approach delivers clearly under-estimated values. At first glance, this under-estimation seems to contradict the over-estimation of the wound's length. The manual wound measurement approaches described in Sec. 5.1 are, however, fully independent, i.e. the manual measurements for length and area do not necessarily correlate. 
Regarding volume measurement, we first have to consider the fact that our volume measurement approach described in Sec. 3.4 uses a bent quadratic surface as estimate for the healthy skin, while the manual reference approach to volume measurement based on the wound phantoms rather refer to a planar wound cap (see Sec. 5.1). Therefore, we additionally estimate the wound's volume with respect to the planar surface fitted to the wound's contour (see Sec. 3.4) for comparison with the manually estimated volume. Still, the comparison is not very expressive, since the manual approach using water filling results in over-estimated volumes as the water's surface can easily exceed the wound contour level due to the water's surface tension. From this perspective, it is not extremely surprising, that our approach "underestimates" the wound's volume if compared to the manual reference.

Comparing the wound measurement for the individual phantoms in Tab. 1, column meas.comp., we realize that the Img_Acc and the Video sequences deliver quite comparable results.

Having a closer look at the Large/Img_Fast experiment, we find that the number of images acquired (13) is significantly lower compared to the other image sequences. While the scale accuracy, the geometry error and the segmentation quality indicate a successful segmentation, our approach could not retrieve the wound's contour properly. This is due to the fact, that the clustering result for the skin region failed to identify a single region, as the number of skin points is very low. Subsequently, the final skin region does not enclose the wound completely and, consequently, the contour has not been extracted properly (see Sec. 3.3 and Fig. 5). Still, if a sufficient number of images is acquired, the Img_Fast method also produces reasonable results, as has been demonstrated by the Img_Fast wound acquisitions for the Medium and the Small phantom. Note that the calculation of the segmentation quality in Tab. 1, column meas.comp., for the Large/Img_Fast experiment accounts for all skin points, not only the main cluster. This, however, does not have any significant impact on the segmentation quality, as the evaluation assumes a binary classifier w/o a miscellaneous class.

\section{Public Science}

We will make all digital data, i.e., the digital models of the wound phantoms, the photographs, and the wound measurement prototype publicly available via GitLab upon publication.

\subsection{Conclusion \& Limitations}

In this paper we present a novel method for 3D measurement of chronic wounds that solely relies on standard RGB imagery. The approach incorporates structure-from-motion as 3D reconstruction, CIELABbased color segmentation of the wound and skin region, wound contour reconstruction, and surface fitting to emulate the healthy skin state. Compared to prior techniques presented in literature, our approach has minimal requirements regarding image acquisition, i.e. standard cameras in mobile devices such as smartphones are sufficient. The quantitative and qualitative evaluation of our approach using realistic wound phantoms and different image acquisition modes yields very robust results, if the number of images acquired is large enough. Ignoring cases of insufficient image counts, all three image acquisition types, i.e. Img_Fast, Img_Acc, and Video, yield good segmentation results and wound measures. A higher number of input images and larger image resolution for the Img_Acc method, however, requires more time to capture and increases computational cost.

The main limitation of our approach is that although we have used statistical approaches to determine thresholds and other parameters wherever possible, there are still some parameters that need to be adjusted manually. This mainly refers to clustering approach [24] and wound segmentation. Furthermore, we so far have not been able to apply our approach to real wounds.

\section{REFERENCES}

[1] M. Augustin and K. Maier. Psychosomatic aspects of chronic wounds. Dermatology and Psychosomatics/Dermatologie und Psychosomatik, 4(1):5-13, 2003.

[2] A. R. Siddiqui and J. M. Bernstein. Chronic wound infection: Facts and controversies. Clinics in Dermatology, 28(5):519-526, 2010.

[3] C. K. Sen, G M. Gordillo, S. Roy, R. Kirsner, L. Lambert, T. K. Hunt, F. Gottrup, G. C. Gurtner, and T. Longaker. Human skin wounds: A major and snowballing threat to public health and the economy. Wound Repair and Regeneration, 17(6):763-771, 2009.

[4] L. Casas, B. Castaneda, and S. Treuillet. Imaging technologies applied to chronic wounds. In Proc. Int. Symp. Applied Sciences in Biomedical and Communication Technologies (ISABEL), pages 1-5. ACM, 2011.

[5] S. Treuillet, B. Albouy, and Y. Lucas. Threedimensional assessment of skin wounds using a standard digital camera. IEEE Transactions on Medical Imaging, 28(5):752-762, 2009.

[6] R. Khoo and S. Jansen. The evolving field of wound measurement techniques: A literature review. Wounds: a compendium of clinical research and practice, 28(6):175, 2016.

[7] E. Sirazitdinova and T. M. Deserno. Introducing low-cost stereo imaging for cutaneous wound as- 
sessment. In Proc. IEEE Symp. Computer-Based Medical Systems, pages 138-139. IEEE, 2016.

[8] A. Gaur, R. Sunkara, A. Raj, and T. Celik. Efficient wound measurements using RGB and depth images. Int. Journal of Biomedical Engineering and Technology, 18(4):333-358, 2015.

[9] D. Bulczak and A. Kolb. Efficient subsurface scattering simulation for time-of-flight sensors. In Proc. Vision, Modeling \& Visualization (VMV), 2018. DOI: 10.2312/vmv.20181259.

[10] M. Kay, J. Santos, and M. Takane. mHealth: New horizons for health through mobile technologies. Global Observatory for eHealth Series, 64(7):6671, 2011.

[11] R. Mukherjee, S. Tewary, and A. Routray. Diagnostic and prognostic utility of non-invasive multimodal imaging in chronic wound monitoring: a systematic review. Journal of Medical Systems, 41(3):46, 2017.

[12] M. Herbin, F.-X. Bon, A. Venot, F. Jeanlouis, M.L. Dubertret, L. Dubertret, and G. Strauch. Assessment of healing kinetics through true color image processing. IEEE Transactions on Medical Imaging, 12(1):39-43, 1993.

[13] S. H. Sprigle, M. Nemeth, and A. Gajjala. Iterative design and testing of a hand-held, non-contact wound measurement device. Journal of tissue viability, 21(1):17-26, 2012.

[14] B. Albouy, S. Treuillet, Y. Lucas, and J C Pichaud. Volume estimation from uncalibrated views applied to wound measurement. In Proc. Int. Conf. Image Analysis and Processing, pages 945-952, 2005.

[15] K. Kolev, P. Tanskanen, P. Speciale, and M. Pollefeys. Turning mobile phones into 3D scanners. In Proc. IEEE Conf. Computer Vision and Pattern Recognition (CVPR), pages 3946-3953, 2014.

[16] O. Muratov, Y. Slynko, V. Chernov, M Lyubimtseva, A Shamsuarov, and V. Bucha. 3DCapture: $3 \mathrm{~d}$ reconstruction for a smartphone. In Proc. IEEE Conf. Computer Vision and Pattern Recognition (CVPR) - Workshops, pages 893-900, 2016.

[17] E. Sirazitdinova and T.M. Deserno. System design for 3D wound imaging using low-cost mobile devices. In SPIE Medical Imaging, page 1013810, 2017.

[18] P. Ondrúška, P. Kohli, and S. Izadi. Mobilefusion: Real-time volumetric surface reconstruction and dense tracking on mobile phones. IEEE Trans. Visualization and Computer Graphics, 21(11):1251-1258, 2015.

[19] J. L. Schönberger and J.-M. Frahm. Structurefrom-motion revisited. In Proc. IEEE Conf. Com- puter Vision and Pattern Recognition (CVPR), pages 4104-4113, 2016.

[20] D. G. Lowe. Distinctive image features from scale-invariant keypoints. International Journal of Computer Vision, 60:91-110, 2004.

[21] B. D. Lucas and T. Kanade. An iterative image registration technique with an application to stereo vision. In Proc. Int. Joint Conf. Artificial Intelligence (IJCAI), pages 674-679, 1981.

[22] B. Triggs, P. F. McLauchlan, R. I. Hartley, and A. W. Fitzgibbon. Bundle adjustment-a modern synthesis. In International workshop on vision algorithms, pages 298-372. Springer, 1999.

[23] S. Garrido-Jurado, R. Muñoz-Salinas, F. J. Madrid-Cuevas, and M. J. Marín-Jiménez. Automatic generation and detection of highly reliable fiducial markers under occlusion. Pattern Recognition, 2014. DOI: 10.1016/j.patcog.2014.01.005.

[24] M. Ester, H.-P. Kriegel, J. Sander, and X. Xu. A density-based algorithm for discovering clusters in large spatial databases with noise. In Proc. Conf. Knowledge Discovery and Data Mining (KDD), volume 96, pages 226-231, 1996.

[25] H. Lee, B. Lee, J. Park, and W. Sun. Segmentation of wounds using gradient vector flow. In Proc. Int. Conf. Intelligent Informatics and Biomedical Sciences (ICIIBMS), pages 390-391, 2015.

[26] G. W. Zack and E. Rogers. Automatic measurement of sister chromatid exchange frequency. The Journal of the Histochemistry and Cytochemistry, 1977. DOI: $10.1177 / 25.7 .70454$.

[27] M. Kazhdan, M. Bolitho, and H. Hoppe. Poisson surface reconstruction. Proc. Symp. Geometry Processing, pages 61-70, 2006.

[28] C. Zhang and T. Chen. Efficient feature extraction for $2 \mathrm{~d} / 3 \mathrm{~d}$ objects in mesh representation. In Proc. Int. Conf. Image Processing, volume 3, pages 935-938, 2001.

[29] J. L. Schönberger, E. Zheng, J.-M. Frahm, and M. Pollefeys. Pixelwise view selection for unstructured multi-view stereo. In Lecture Notes in Computer Science, volume 9907, pages 501-518, 2016.

[30] T. E. Oliphant. SciPy: Open source scientific tools for python. Computing in Science and Engineering, 2007. DOI: 10.1109/MCSE.2007.58.

[31] W. McKinney. Data structures for statistical computing in python. In Proc. Conf. Python in Science, volume 445, pages 51-56, 2010.

[32] S. van der Walt, J. L. Schönberger, J NunezIglesias, F. Boulogne, J.D. Warner, N. Yager, E. Gouillart, and T. Yu. scikit-image: Image processing in python. PeerJ, 2:e453, 2014. 